\title{
Exact Solution of an MHD Natural Convection Flow in Vertical Concentric Annulus with Heat Absorption
}

\author{
Yusuf Samuel Taiwo \\ Department of Mathematics, Ahmadu Bello University, Zaria, Nigeria \\ Email address: \\ taiyeee@yahoo.com \\ To cite this article: \\ Yusuf Samuel Taiwo. Exact Solution of an MHD Natural Convection Flow in Vertical Concentric Annulus with Heat Absorption. \\ International Journal of Fluid Mechanics \& Thermal Sciences. Vol. 3, No. 5, 2017, pp. 52-61. doi: 10.11648/j.ijfmts.20170305.12
}

Received: March 8, 2017; Accepted: March 30, 2017; Published: November 28, 2017

\begin{abstract}
This paper presents an exact solution of a fully developed natural convection flow in a vertical concentric annulus in the presence of transverse magnetic field and heat absorption. The non-dimensional form of the equation governing the flow is first obtained and then the unified analytical solutions for the temperature field, velocity field, and skin-frictions as well as rate of heat transfer are obtained for both isothermal and constant heat flux case on the outer surface of the inner cylinder. The effect of various identified governing parameters on the flow was illustrated with the aid of line graphs. It is found that the magnitude of maximum fluid velocity is greater in the case of isothermal heating compared with the constant heat flux case when the gap between the cylinders is less or equal to radius of the inner cylinder. More also, the various values of the nondimensional heat absorption parameter $(\mathrm{H})$ and the corresponding values of annular gap where these fields are almost the same are presented in table 1 .
\end{abstract}

Keywords: MHD, Natural Convection, Annulus, Heat Absorption, Isothermal, Isoflux

\section{Introduction}

Interest in buoyancy-driven flows and the related heat transfer has been on increase over the past few decades because of the importance of these flows in many different areas (such as cooling of electronic equipment, pollution, materials processing, energy systems and safety in thermal processes). The subject has been extensively discussed by a number of books, for example, Kakac et al. [1], Bejan and Kraus [2] and Turner [3]. The study of heat generation or absorption in working fluid is important in problems dealing with chemical reactions and those concerned with fluid dispersion. Effect of heat absorption may vary the temperature profiles and this, in turn, can affect the rate of deposition of particles in nuclear reactors and electronic chips, this singular fact has aroused the interest of several researchers. Although exact modeling of internal heat generation or absorption is quite difficult, some simple mathematical models can be used to express its general behaviour for most physical situations. Heat generation or absorption can be assumed to be constant this can be found in the work of Inman [4], it was taken to be space-dependent in the work of Chambre [5], several works have also shown that it can be expressed as a function of temperature Modejski [6] and Toor [7]. The use of magnetic field that influences heat generation/absorption process in electrically conducting fluid flows has many engineering applications. For example, many metallurgical processes which involve cooling of continuous strips, which are drawn through a quiescent fluid. It is a known fact that in a physical application such as crystal growing the heat generation or absorption effect in the fluid is greatly dependent on temperature. Vajravelu and Nayfeh [8] shows this dependence by a linear relationship. Miyatake and Fujii [9] have discussed the free convection flow between vertical plates - one plate isothermally heated and other thermally insulated. Natural convection flow between vertical parallel plates- one plate with a uniform heat flux and the other thermally insulated have been investigated by Tanaka et al. [10]. Jha and Ajibade [11] have studied the unsteady free convective Couette flow of heat generating/absorbing fluid. They have also examined the effect of periodic heat input in fluid flowing in a vertical porous plate [12]. Das et al. [13] studied the radiation effects on free convection MHD Couette flow started exponentially with variable wall temperature in presence of heat generation. The effect of radiation on transient natural 
convection flow between two vertical walls have been described by Mandal et al. [14]. Das et al. [15] have studied the radiation effects on unsteady MHD free convective Couette flow of heat generating/absorbing fluid. Chamkha [16] made an analysis of mixed convection in a square cavity in the presence of the magnetic field and internal heat generation or absorption, he obtained that the magnetic field largely affected the behaviour and the heat transfer characteristics within the cavity. Singh et al. [17] have investigated the effect of mixed kind of thermal boundary conditions on the free convection flow of an electrically conducting fluid in annulus under the influence of a radial magnetic field. Kalita [18] has obtained an exact solution of an unsteady free convection MHD flow and heat transfer between two heated vertical plates with heat source. In a related work Singh and Singh [19] studied the effect of induced magnetic field on natural convection in vertical concentric annulus and concluded that the magnitude of maximum value of the fluid velocity as well as induced magnetic field is greater in the case of isothermal condition compared with the constant heat flux case when the gap between the cylinders is less or equal to 1.70 times the radius of inner cylinder, while reverse trend occurs when the gap between the cylinders is greater than 1.71 times the radius of inner cylinder. Other related article is the work of Kumar [20]. The aim of the present paper is to perform a study of MHD natural convection flow in a vertical concentric annulus with heat absorption. The unified solutions for the temperature, velocity, skin friction and mass flow rate in non-dimensional form with mixed kind thermal boundary condition have been obtained.

\section{Mathematical Analysis}

We have considered here, steady laminar fully developed natural convective flow of an electrically conducting fluid in the vertical concentric annulus of infinite length. The $z^{\prime}$-axis is taken along the axis of the cylinder in the vertically upward direction opposite the gravitational force. In the present physical situation, the inner cylinder is heated or cooled either isothermally or at a constant heat flux. While the inner surface of the outer cylinder is kept at $T_{0}$. Since the flow is fully developed the flow depends only on radial coordinate $r^{\prime}$. A schematic diagram of the problem is shown in Figure 1. Thus under the usual Boussinesq approximation, the basic governing equation for the model under consideration in dimensional form are given by;

$$
\begin{gathered}
v\left[\frac{d^{2} U^{\prime}}{\partial r^{\prime 2}}+\frac{1}{r^{\prime}} \frac{d U^{\prime}}{d r^{\prime}}\right]-\frac{\sigma B_{0}}{\rho} U^{\prime}+g \beta\left(T^{\prime}-T_{0}\right)=0 \\
\frac{k}{\rho c_{p}}\left[\frac{d^{2} T^{\prime}}{d r^{\prime 2}}+\frac{1}{r^{\prime}} \frac{d T^{\prime}}{d r^{\prime}}\right]-\frac{Q_{0}}{\rho c_{p}}\left(T^{\prime}-T_{0}\right)=0
\end{gathered}
$$

The boundary conditions suggested by the physics of the problem are:

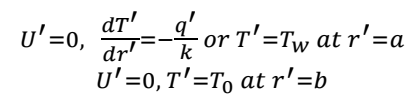

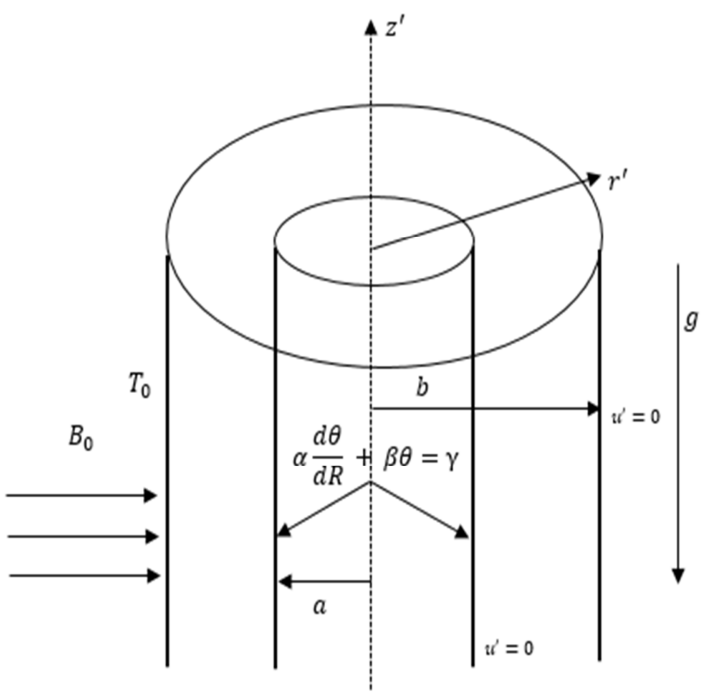

Figure 1. Flow configuration and coordinates system.

The dimensional quantities in the momentum equation, energy equation including the boundary condition (3) above are defined in the nomenclature

Introducing the following non-dimensional quantities:

$$
\begin{gathered}
R=\frac{r^{\prime}}{a}, \lambda=\frac{b}{a}, H^{2}=\frac{a^{2} Q_{0}}{k}, \theta=\frac{\left(T^{\prime}-T_{0}\right)}{\Delta T}, \operatorname{Pr}=\frac{\mu c_{p}}{k} \\
U=\frac{U^{\prime}}{U_{0}}, U_{0}=\frac{g \beta\left(T_{w}-T_{0}\right) a^{2}}{v}, M^{2}=\frac{\sigma B_{0}{ }^{2} a^{2}}{\rho v}
\end{gathered}
$$

Where $\Delta T=T_{w}-T_{0}$ or $\frac{q^{\prime} a}{r^{\prime}}$ according as inner cylinder is maintained at constant temperature $T_{w}$ or constant heat flux $q^{\prime}$, respectively.

Using equation (4), the non-dimensional expression for momentum and energy equation are

$$
\begin{gathered}
{\left[\frac{d^{2} U}{d R^{2}}+\frac{1}{R} \frac{d U}{d R}\right]-M^{2} U=-\theta} \\
\frac{d^{2} \theta}{d R^{2}}+\frac{1}{R} \frac{d \theta}{d R}-H^{2} \theta=0
\end{gathered}
$$

While the boundary conditions in dimensionless form are given as:

$$
\begin{gathered}
U=0 \frac{d \theta}{d R}=-1 \text { or } \theta=1 \text { at } R=1 \\
U=0 \theta=0 \text { at } R=\lambda
\end{gathered}
$$

The isothermal and heat flux boundary condition as given in equation (7) can be represented by a single equation as

$$
\alpha \frac{d \theta}{d R}+\beta \theta=\gamma \text { at } R=1
$$

In which the suitable values of $\alpha, \beta$ and $\gamma$ represent the isothermal or isoflux condition at the outer surface of the inner cylinder as the case maybe.

The solution of equation (5) and (6) subject to the boundary conditions (8) and (9) are;

$$
U=C_{3} I_{0}(R M)+C_{4} K_{0}(R M)-\frac{\left[C_{1} I_{0}(R H)+C_{2} K_{0}(R H)\right]}{\left[H^{2}-M^{2}\right]}
$$




$$
\theta=C_{1} I_{0}(R H)+C_{2} K_{0}(R H)
$$

Where $C_{1}, C_{2}, C_{3}, C_{4}$ are defined in the appendix

$$
\begin{gathered}
\tau_{1}=\left.\frac{d U}{d R}\right|_{R=1}=M\left(C_{3} I_{1}(M)-C_{4} K_{1}(M)\right)-\frac{H\left[C_{1} I_{1}(H)-C_{2} K_{1}(H)\right]}{\left[H^{2}-M^{2}\right]} \\
\tau_{\lambda}=-\left.\frac{d U}{d R}\right|_{R=\lambda}=M\left(C_{4} K_{1}(\lambda M)-C_{3} I_{1}(\lambda M)\right)+\frac{H\left[C_{1} I_{1}(\lambda H)-C_{2} K_{1}(\lambda H)\right]}{\left[H^{2}-M^{2}\right]}
\end{gathered}
$$

Also, the mass flow rate in of fluid through the annular region is

$$
Q=2 \pi \int_{1}^{\lambda} R U d R=\frac{C_{3}}{M}\left[\lambda I_{1}(\lambda M)-I_{1}(M)\right]-\frac{C_{4}}{M}\left[\lambda K_{1}(\lambda M)-K_{1}(M)\right]-\frac{\left[C_{1}\left\{\lambda I_{1}(\lambda H)-I_{1}(H)\right\}-C_{2}\left\{\lambda K_{1}(\lambda H)-K_{1}(H)\right\}\right]}{H\left[H^{2}-M^{2}\right]}
$$

Using equation (11), the rate of heat transfer at both surfaces are respectively given by

$$
\begin{gathered}
N u_{1}=-\left.\frac{d \theta}{d R}\right|_{R=1}=H\left(C_{2} K_{1}(H)-C_{1} I_{1}(H)\right) \\
N u_{\lambda}=-\left.\frac{d \theta}{d R}\right|_{R=\lambda}=H\left(C_{2} K_{1}(\lambda H)-C_{1} I_{1}(\lambda H)\right)
\end{gathered}
$$

\section{Results and Analysis}

In other to have a good knowledge of the effects of the governing parameters on the fluid temperature profiles, velocity profiles, skin-friction and rate of heat transfer at both surfaces as well as mass flow rate in the annular gap, we have employed a mathematical tool- MATLAB to generate the numerical values and line graph of the temperature $(\theta)$, velocity $(U)$, skin-friction $\left(\tau_{1}\right)$ at the outer surface of the inner cylinder, skin-friction $\left(\tau_{\lambda}\right)$ at the inner surface of the outer cylinder, Nusselt number $\left(N u_{1}\right)$ at the outer surface of the inner cylinder, Nusselt number $\left(N u_{\lambda}\right)$ at the inner surface of the outer cylinder and mass flow rate $(\mathrm{Q})$ for some carefully selected flow parameters so as to comment on their effects on the flow formation.

Figure 2 points out the temperature profiles across the annular gap for different values of the dimensionless heat absorption parameter $(H)$ for both isothermal and isoflux heating. We can see that the fluid temperature decreases with increase in $H$, it is also observed to decrease towards the inner surface of the outer cylinder. In addition, fluid temperature due to isothermal heating is observed to be higher than the heat flux case.

The velocity for isothermal and constant heat flux are shown in Figures $3-6$. It can be seen from Figure 3 that velocity decreases with increase in heat absorption parameter $(\mathrm{H})$, but seen to be inversely proportional to the radii ratio in both cases, higher magnitude of velocity is also observed to have occurred in the isothermal case. Figures 4, 5 and 6 show variation of velocity profiles for different values of Hartmann number $(\mathrm{M})$. We observed in these Figures that increase in Hartmann number decreases the fluid velocity however, it is clear from Figures 4 and 5 that the magnitude of maximum value of the velocity profile is higher in the case of isothermal condition than the case of constant heat flux when the radius of outer cylinder is less than $3.5(\lambda<3.5)$ while reverse phenomena occur when $\lambda>3.5$. Again, it is also clear from Figure 6 that the velocity profiles for $\lambda=3.5$ are approximately the same for both cases of thermal conditions imposed on the inner cylinder. Other corresponding values of $\lambda$ and $\mathrm{H}$ where velocity profiles are approximatively the same for both isothermal and constant heat flux are presented in table 1 .

Figures 7 to 10 , present the skin friction $\left(\tau_{1}\right)$ at the outer surface of the inner cylinder and the skin friction $\left(\tau_{\lambda}\right)$ at the inner surface of the outer cylinder for different controlling parameters. In Figures 7 and 8. We obtained that both $\tau_{1}$ and $\tau_{\lambda}$ decreases with increase in Hartmann number $(M)$ the Figures also show that the skin friction at both surfaces is inversely proportional to the heat absorption parameter $(\mathrm{H})$ with skin friction due to isothermal heating higher than the heat flux case at both surfaces. The variation of skin friction for different values of $\lambda$ at the outer surface of the inner cylinder and at the inner surface of the outer cylinder is respectively presented by Figures 9 and 10. Figure 9 shows that skin friction $\left(\tau_{1}\right)$ increases with increase in $\lambda$, while Figure 10 shows that skin friction $\left(\tau_{\lambda}\right)$ decreases with increase in $\lambda$. The mass flow rate through the annular gap is presented in Figures 11 to 14. As expected, the Hartmann number always tend to reduce the mass flow rate (See Figures 11 and 12) generally, the mass flow rate (Q) is clearly seen to decrease for $H<1.5$ but increase for values of $H>1.5$ when radii ratio $\lambda=2$.

Figures 13 and 14 present variation of mass flow rate for different values of radii ratio $(\lambda)$. It is observed in Figure 13 that mass flow rate increase with increase in radii ratio $(\lambda)$ the reserve is however seen for large value of heat absorption parameter $(H)$ (See Figure 14).

Figures 15 and 16 depict the rate of heat transfer for different values of $(\lambda)$ and $(H)$. In Figure 15, it is obvious that heat transfer at the outer surface of the inner cylinder when the cylinder is isothermally heated decreases with increase in $\lambda$ but increases with increase in $H$ but maintained a constant value for the heat flux case at the outer surface of the inner cylinder. The rate of heat transfer is observed to decrease with increase in $\lambda$ and the heat absorption parameter (H). (See Figure 16)

\section{Conclusion}

A theoretical study of the effect of Hartmann number and heat absorption parameter, on a steady MHD natural convection flow in vertical concentric annulus with heat 
absorption with the outer surface of the inner cylinder subjected to a mixed kind boundary condition (isothermal and isoflux). We found that Hartmann number (M), heat absorption parameter $(\mathrm{H})$, as well as annular gap, play a major role in determining the nature of fluid flow. The main conclusions of the present research are;

a) The magnitude of maximum value of the velocity profile is higher in the case of isothermal condition than the constant heat flux when the radius of outer cylinder is less than $3.5(\lambda<3.5)$ while reverse phenomena occur when $(\lambda>3.5)$.

b) The velocity profiles for $(\lambda=3.5)$ are approximately the same for both cases of thermal conditions imposed on the inner cylinder. Other corresponding values of $\lambda$ and $\mathrm{H}$ where velocity profiles are approximatively the same are presented in table 1.

c) The skin friction is seen to generally decrease with increase in the heat absorption parameter $(H)$ while the mass flow rate $(Q)$ is clearly seen to decrease for values of $H<1.5$ but increases for $H>1.5$ when radii ratio $(\lambda=2)$.

d) Increase in the annular gap $(\lambda)$ enhances rate of heat transfer at the outer surface of the inner cylinder the reverse is seen at the inner surface of the outer cylinder.

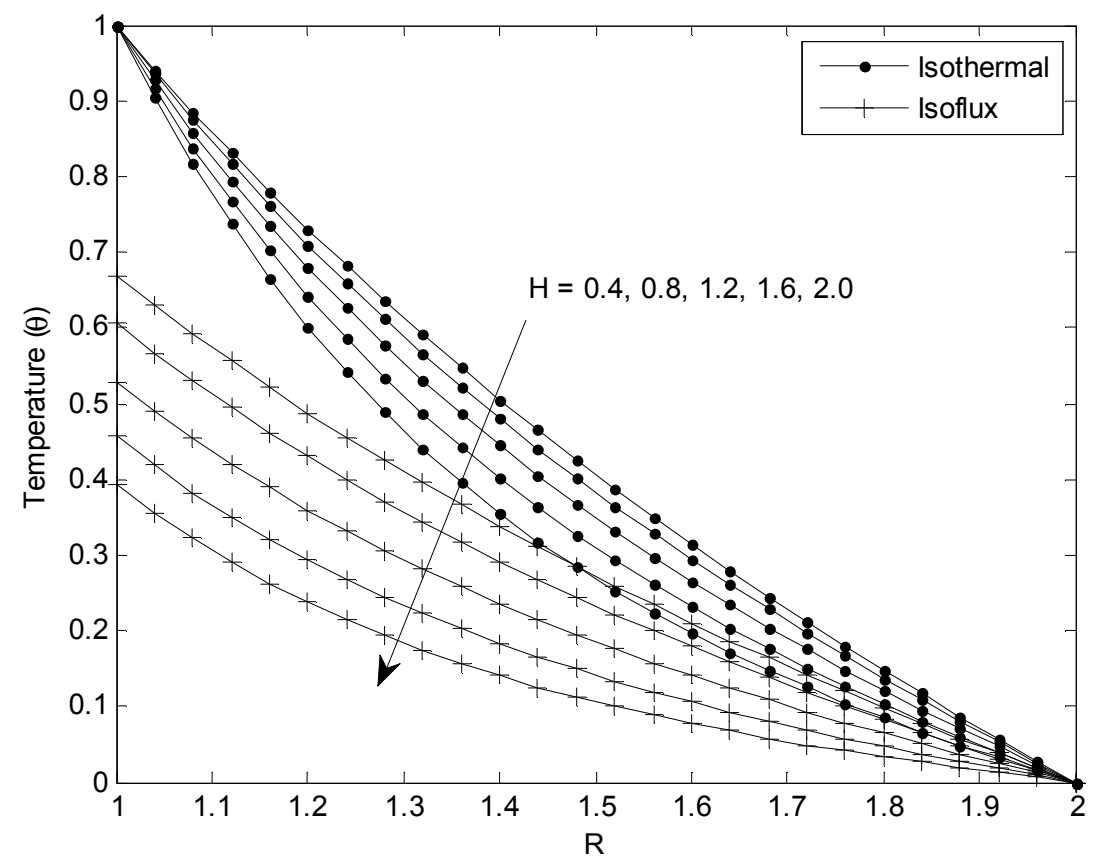

Figure 2. Temperature profiles for different values of $H$ (Isothermal and Isoflux case).

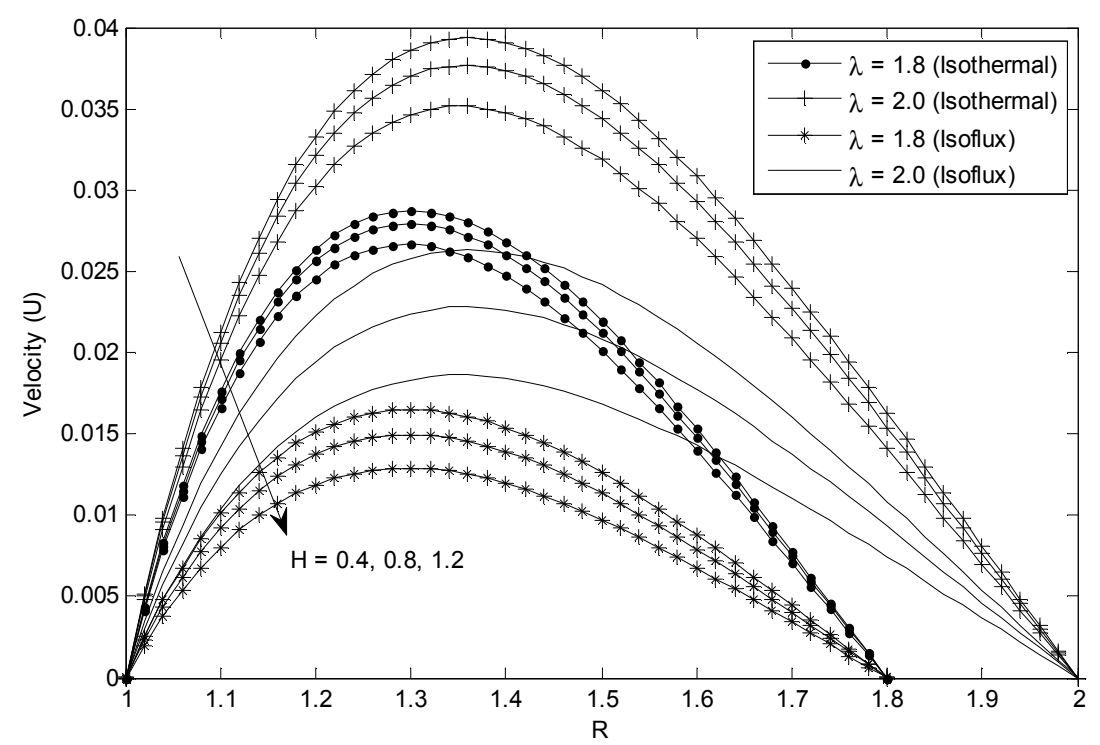

Figure 3. Distribution of velocity components versus $R$ for different values of $H$ when $\lambda=1.8$ and $2(M=2)$. 


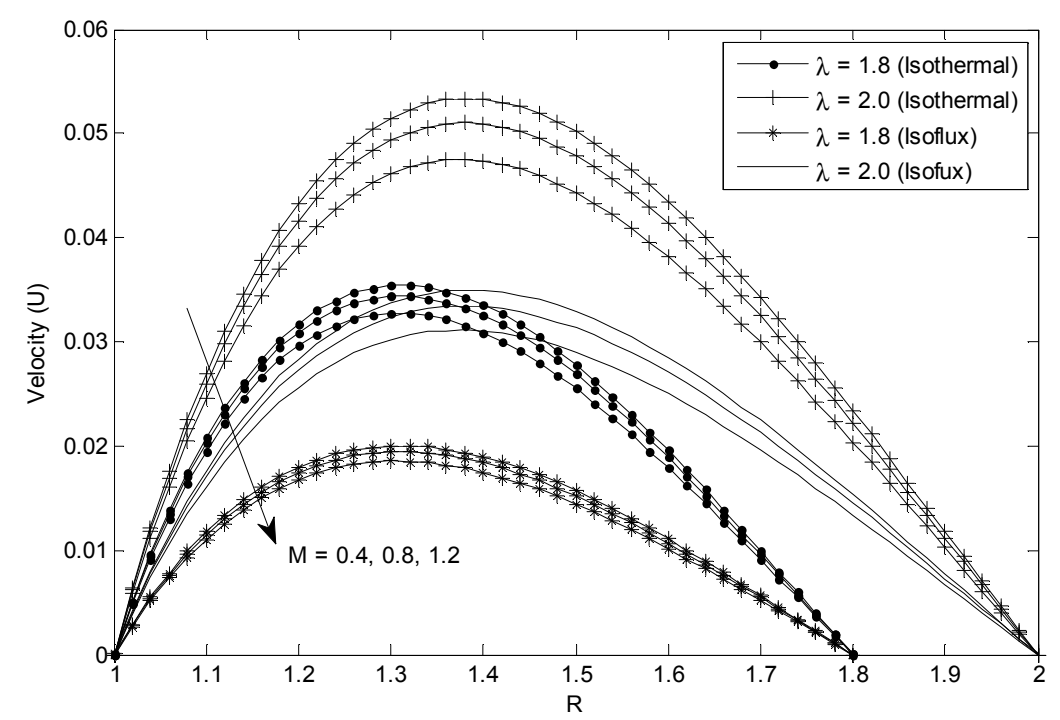

Figure 4. Distribution of velocity components versus $R$ for different values of $M \lambda<3.5(H=0.5)$.

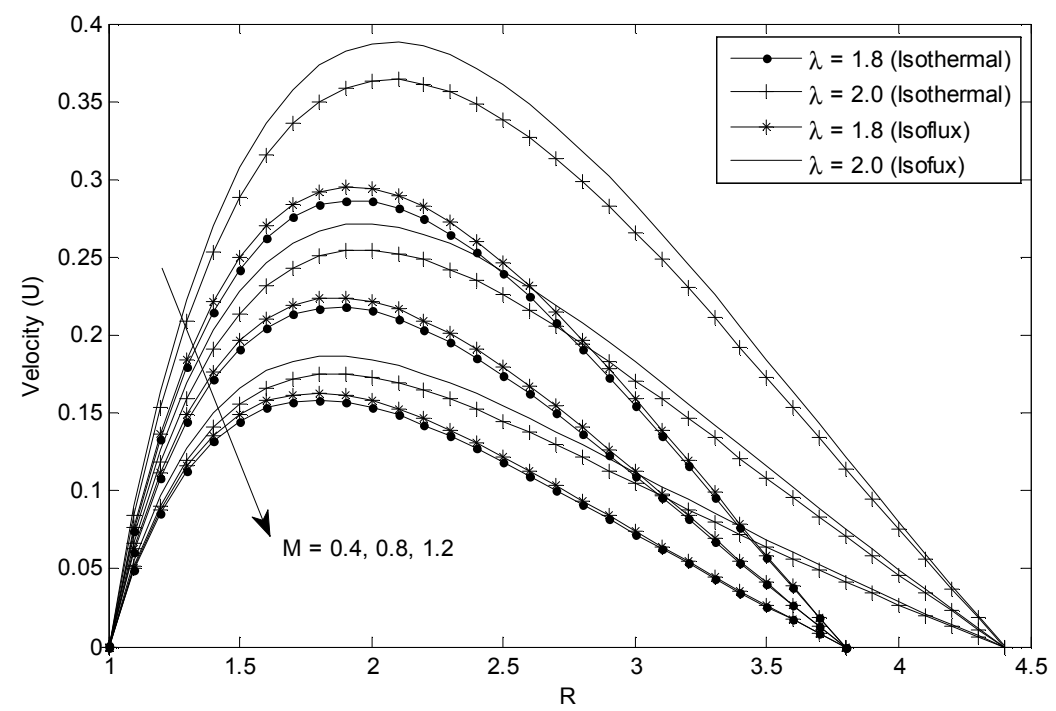

Figure 5. Distribution of velocity components versus $R$ for different values of $M(\lambda>3.5, H=0.5)$.

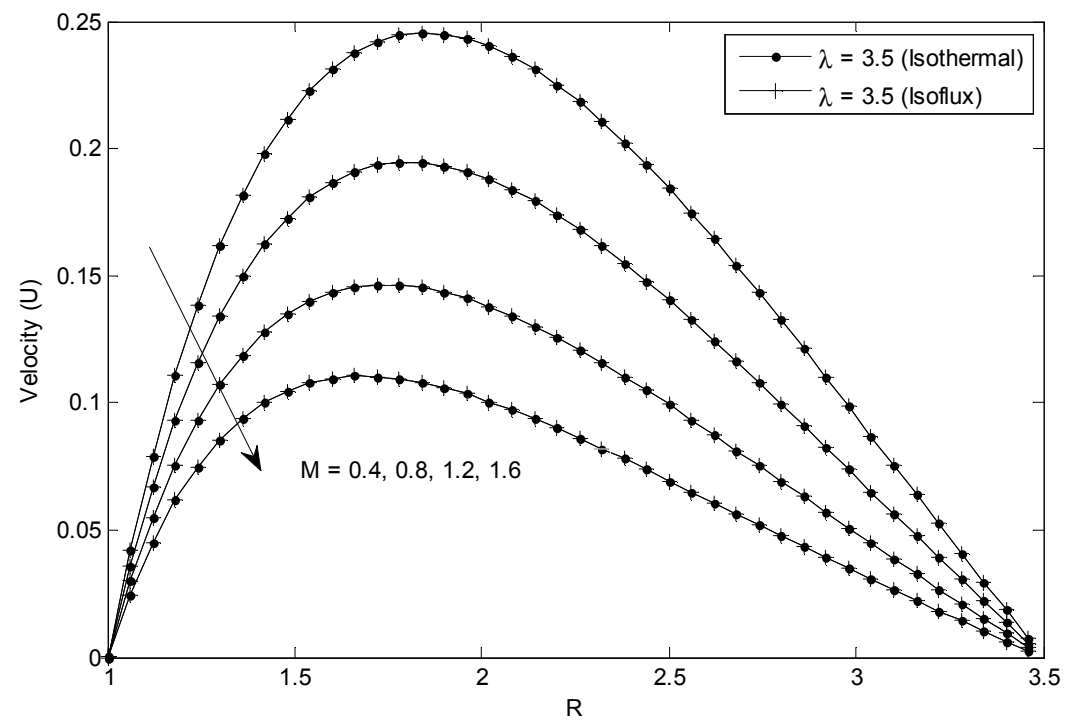

Figure 6. Distribution of velocity components versus $R$ for different values of $M$ when $(H=0.5, \lambda=3.5)$. 


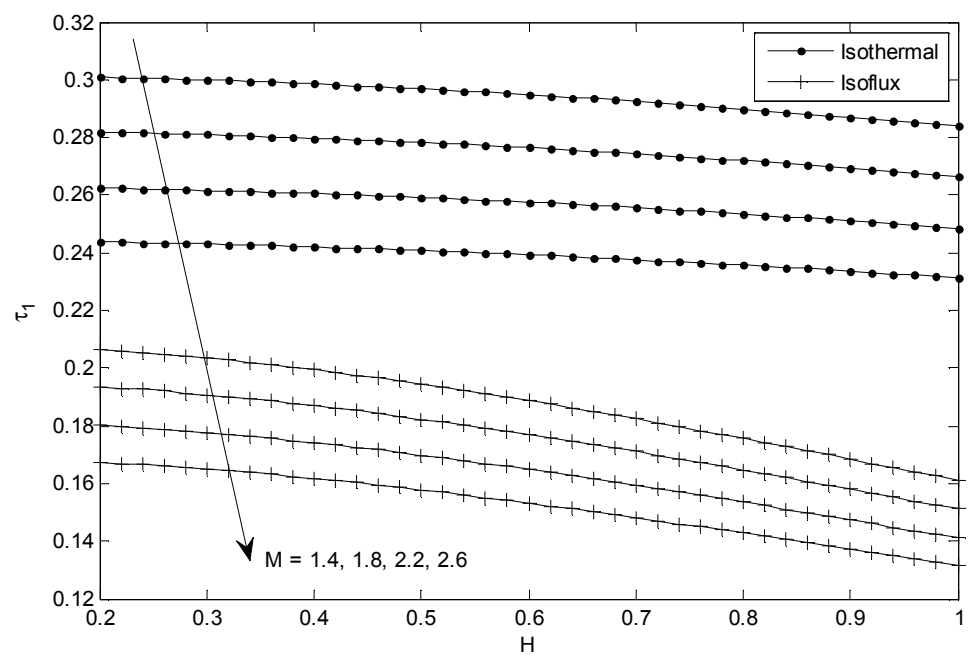

Figure 7. Skin friction at the outer surface of the inner cylinder versus $H$ for different values of $M(\lambda=2)$.

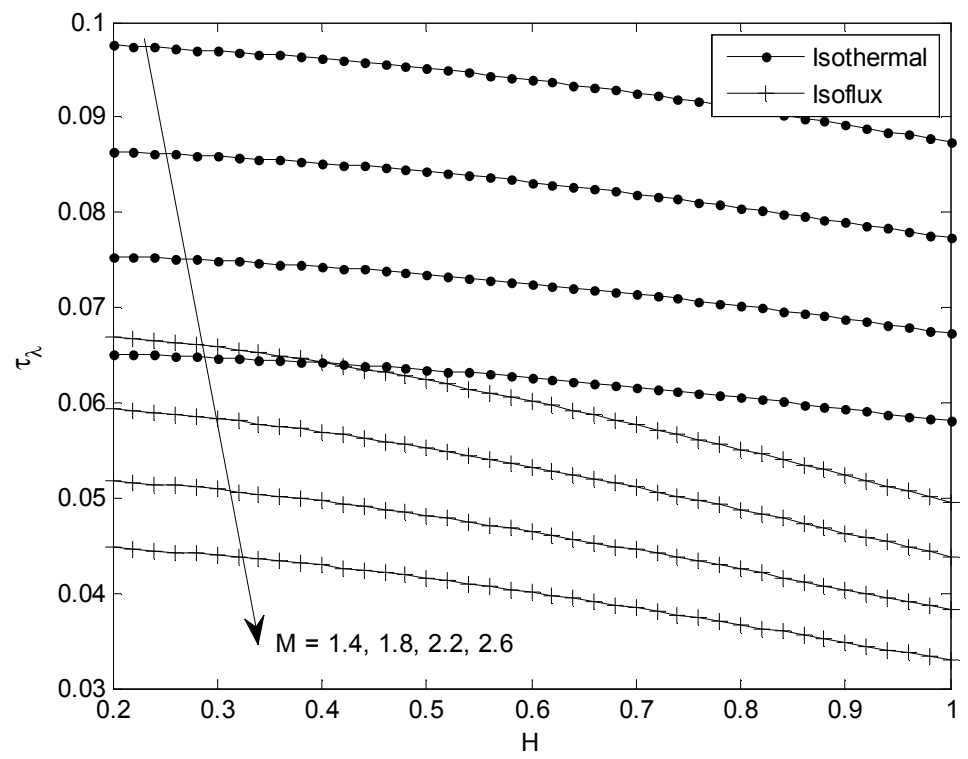

Figure 8. Skin friction at the inner surface of the outer cylinder versus $H$ for different values of $M(\lambda=2)$.

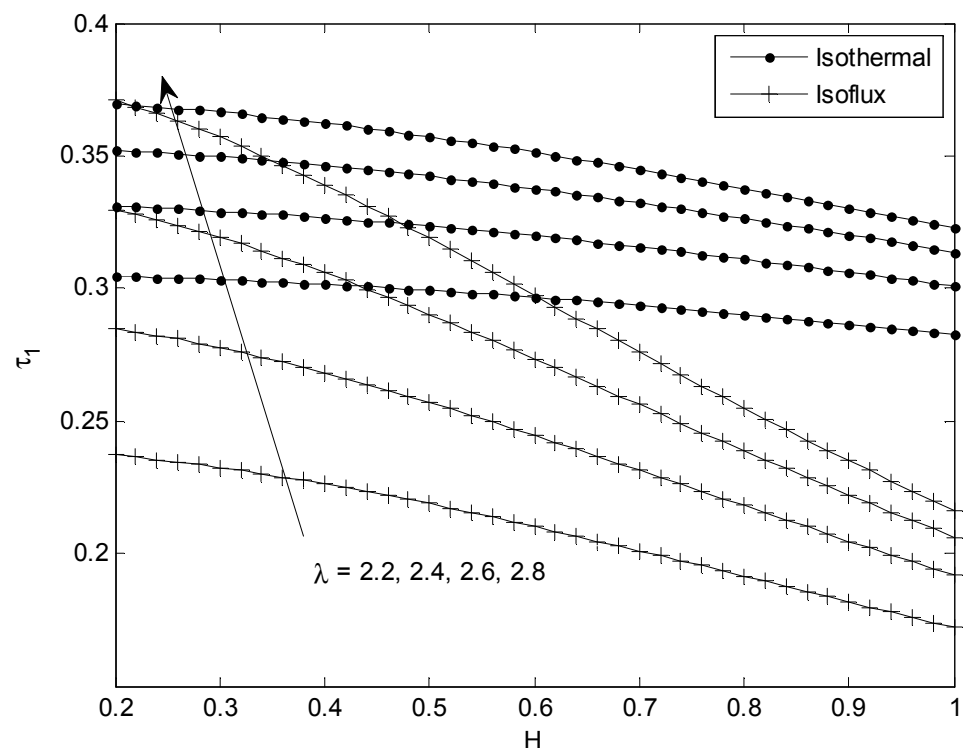

Figure 9. Skin friction at the outer surface of the inner cylinder versus $H$ for different values of $\lambda(M=2)$. 


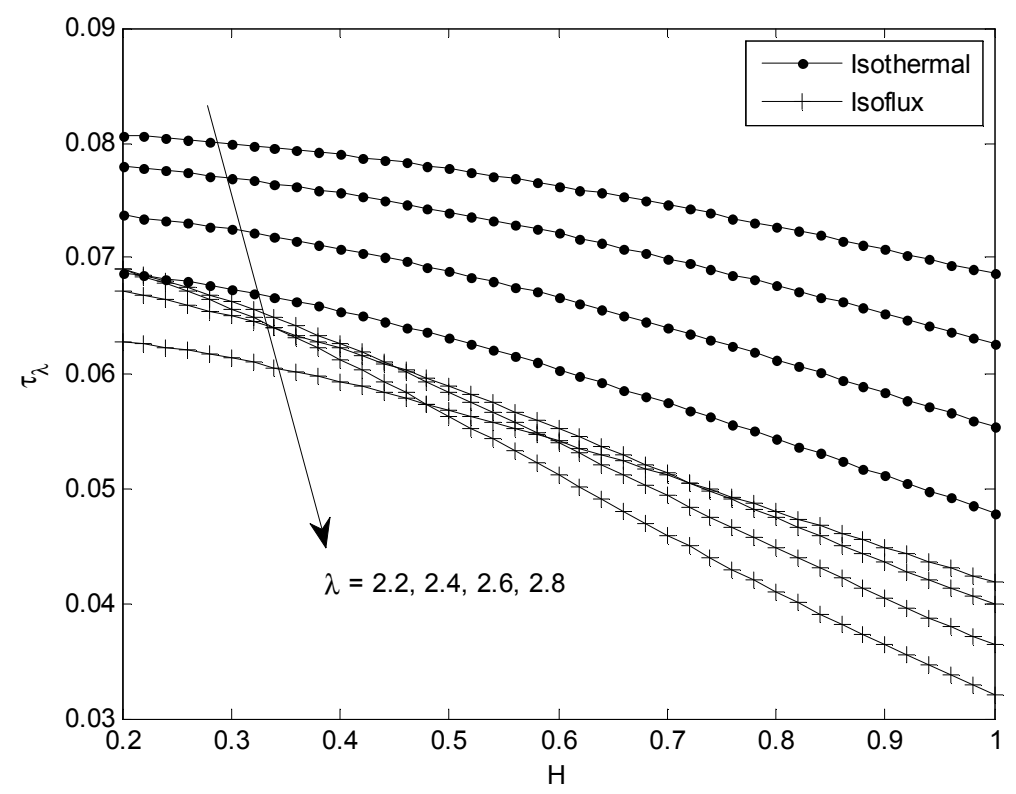

Figure 10. Skin friction at the inner surface of the outer cylinder versus $H$ for different values of $\lambda(M=2)$.

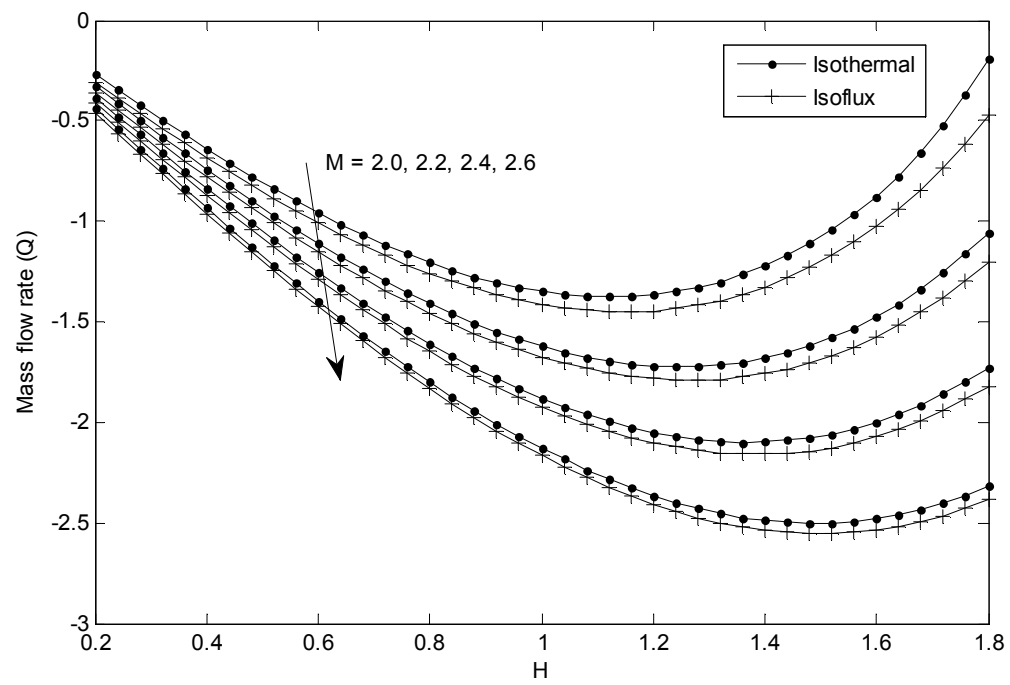

Figure 11. Distribution of Mass flow rate versus $H$ for different values of $M$ for $H<M(\lambda=2.0)$.

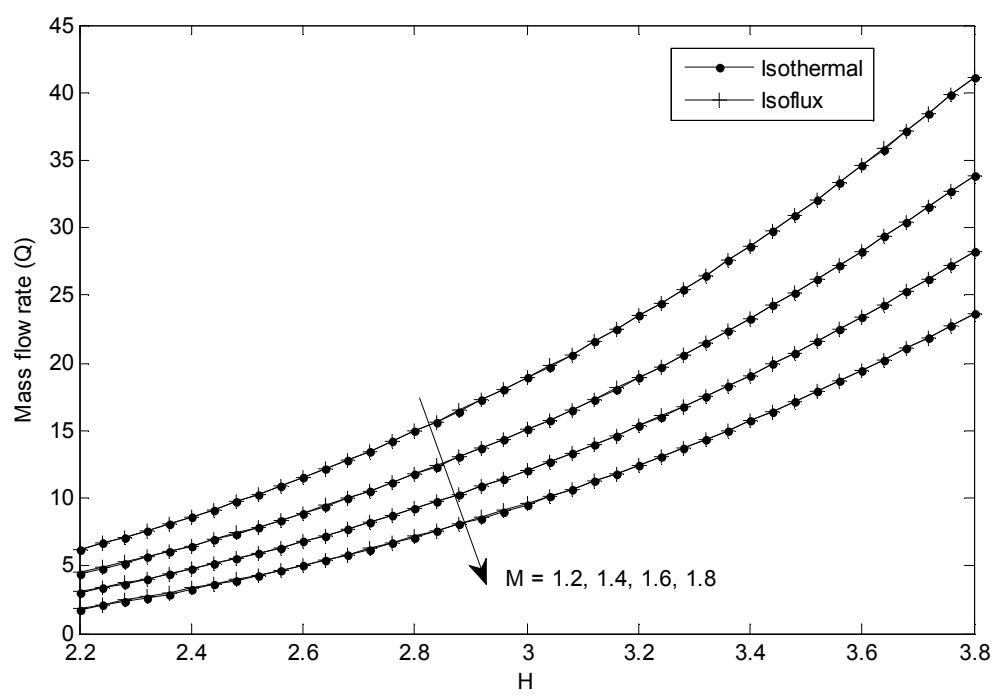

Figure 12. Distribution of Mass flow rate versus $H$ for different values of $M$ for $M<H(\lambda=2.0)$. 


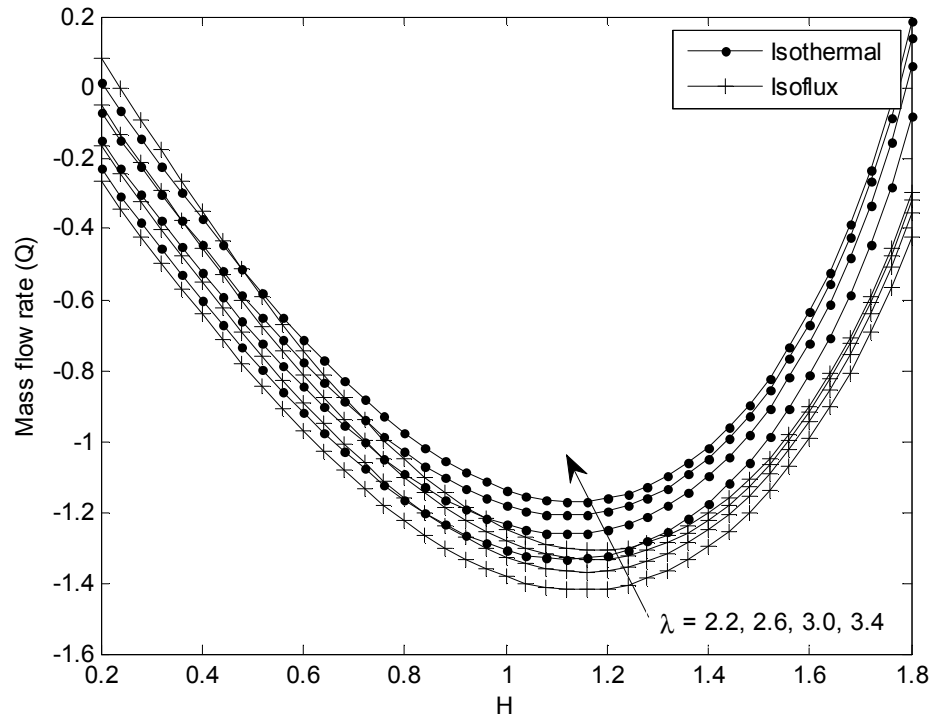

Figure 13. Distribution of Mass flow rate versus $H$ for different values of $\lambda(M>H, M=2.0)$.

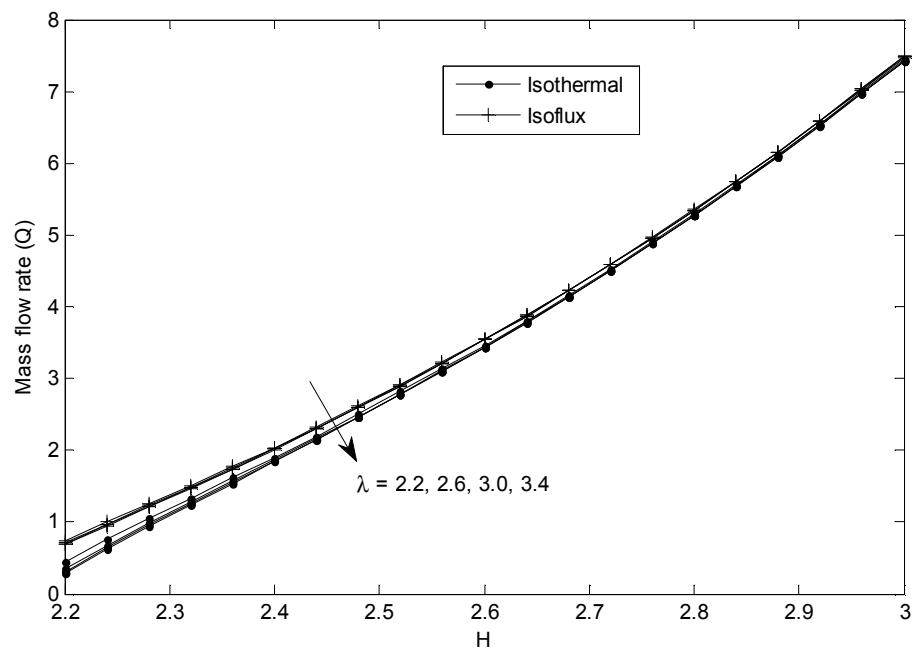

Figure 14. Distribution of Mass flow rate versus $H$ for different values of $\lambda$ for $M<H(M=2.0)$.

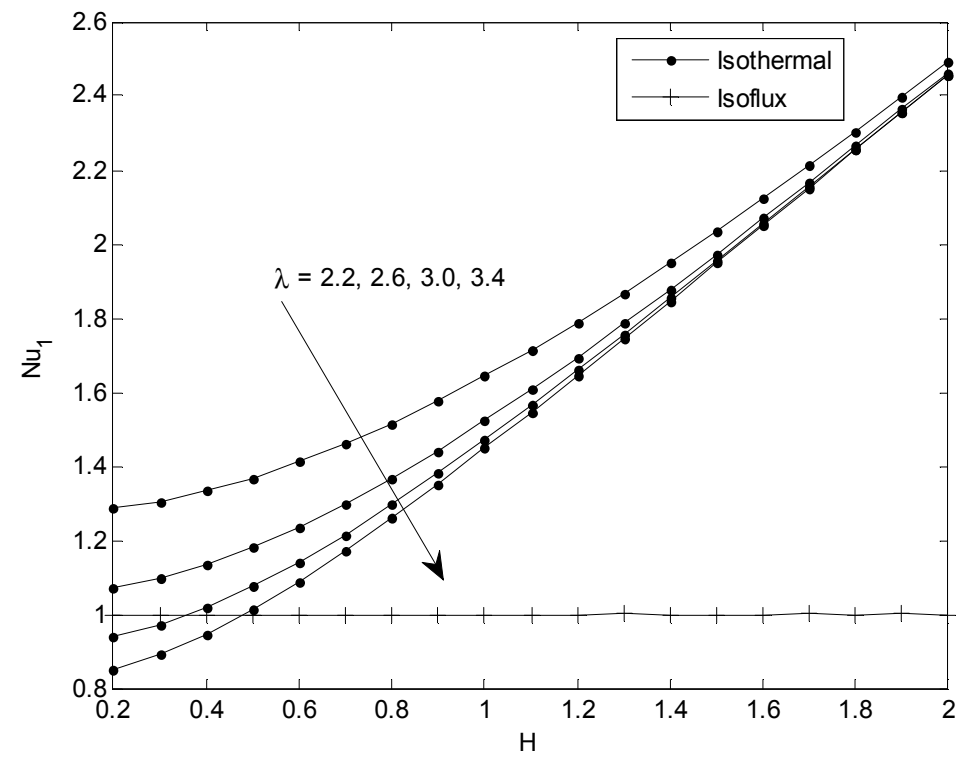

Figure 15. Distribution of rate of heat transfer at the outer surface of the inner cylinder for different values of $H$ and $\lambda$. 


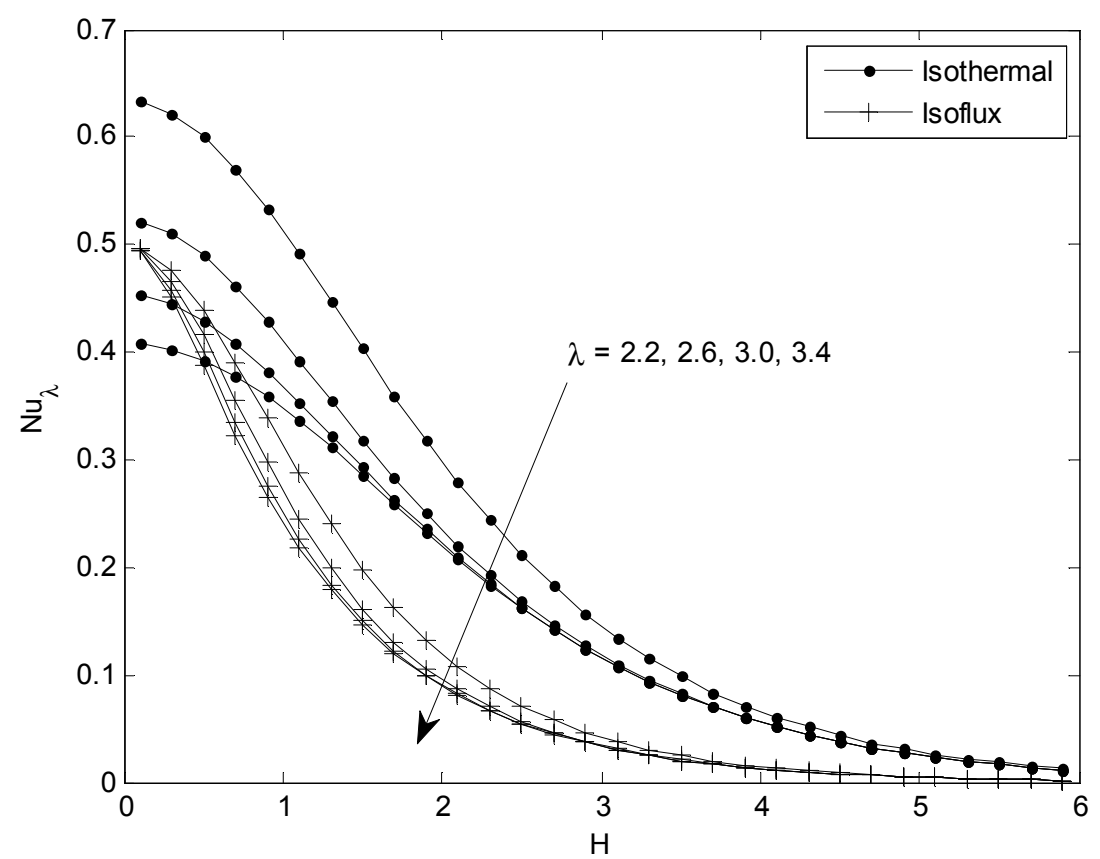

Figure 16. Distribution of rate of heat transfer at the inner surface of the outer cylinder for different values of $H$ and $\lambda$.

Table 1. Showing the corresponding values of $H$ and $\lambda$ for which the fluid velocity for isothermal heating and constant heat flux.

\begin{tabular}{lll}
\hline $\mathbf{H}$ & $\lambda$ & Annular gap \\
\hline 0.01 & 2.7184 (Singh and Singh) & 1.7184 (Singh and Singh) \\
0.04 & 2.7209 & 1.7209 \\
0.08 & 2.7288 & 1.7288 \\
0.10 & 2.7348 & 1.7348 \\
0.20 & 2.7880 & 1.7880 \\
0.30 & 2.8917 & 1.8917 \\
0.40 & 3.0837 & 2.0837 \\
0.50 & 3.5004 & 2.5004 \\
0.59 & 5.7178 & 4.7178 \\
\hline
\end{tabular}

Table 2. Table of convergence of the present work in comparison with the work of Singh and Singh for different values of $H$.

\begin{tabular}{|c|c|c|c|c|c|c|c|c|}
\hline \multirow[b]{3}{*}{$\mathbf{H}$} & \multicolumn{4}{|l|}{$N u_{1}$} & \multicolumn{4}{|l|}{$N u_{\lambda}$} \\
\hline & \multicolumn{2}{|l|}{ Isothermal } & \multicolumn{2}{|l|}{ Isoflux } & \multicolumn{2}{|l|}{ Isothermal } & \multicolumn{2}{|l|}{ Isoflux } \\
\hline & $\begin{array}{l}\text { Singh and } \\
\text { Singh }\end{array}$ & $\begin{array}{l}\text { Present } \\
\text { work } \\
\end{array}$ & Singh and Singh & Present work & $\begin{array}{l}\text { Singh and } \\
\text { Singh }\end{array}$ & $\begin{array}{l}\text { Present } \\
\text { work }\end{array}$ & Singh and Singh & $\begin{array}{l}\text { Present } \\
\text { work }\end{array}$ \\
\hline 2.00 & -1.4427 & -2.5337 & -1.000 & -1.0000 & -0.7213 & -0.3962 & -0.5000 & -0.1564 \\
\hline 1.60 & -1.4427 & -2.1907 & -1.000 & -1.0000 & -0.7213 & -0.4845 & -0.5000 & -0.2212 \\
\hline 1.20 & -1.4427 & -1.8898 & -1.000 & -1.0000 & -0.7213 & -0.5725 & -0.5000 & -0.3029 \\
\hline 0.80 & -1.4427 & -1.6512 & -1.000 & -1.0000 & -0.7213 & -0.6493 & -0.5000 & -0.3932 \\
\hline 0.40 & -1.4427 & -1.4965 & -1.000 & -1.0000 & -0.7213 & -0.7023 & -0.5000 & -0.4693 \\
\hline 0.20 & -1.4427 & -1.4562 & -1.000 & -1.0000 & -0.7213 & -0.7165 & -0.5000 & -0.4920 \\
\hline 0.08 & -1.4427 & -1.4449 & -1.000 & -1.0000 & -0.7213 & -0.7206 & -0.5000 & -0.4987 \\
\hline 0.04 & -1.4427 & -1.4432 & -1.000 & -1.0000 & -0.7213 & -0.7212 & -0.5000 & -0.4997 \\
\hline 0.02 & -1.4427 & -1.4428 & -1.000 & -1.0000 & -0.7213 & -0.7213 & -0.5000 & -0.4999 \\
\hline 0.01 & -1.4427 & -1.4427 & -1.000 & -1.0000 & -0.7213 & -0.7213 & -0.5000 & -0.5000 \\
\hline
\end{tabular}

\section{Appendix}

$$
\begin{aligned}
& C_{1}=\frac{-\gamma K_{0}(\lambda H)}{I_{0}(\lambda H)\left[\beta K_{0}(H)-\alpha H K_{1}(H)\right]-K_{0}(\lambda H)\left[\beta K_{0}(H)-\alpha H K_{1}(H)\right]} \\
& C_{2}=\frac{\gamma I_{0}(\lambda H)}{I_{0}(\lambda H)\left[\beta K_{0}(H)-\alpha H K_{1}(H)\right]-K_{0}(\lambda H)\left[\beta K_{0}(H)-\alpha H K_{1}(H)\right]} \\
& C_{3}=\frac{K_{0}(\lambda M)\left[\gamma K_{0}(\lambda H) I_{0}(H)-\gamma I_{0}(\lambda H) K_{0}(H)\right]}{I_{0}(\lambda H)\left[\beta K_{0}(H)-\alpha H K_{1}(H)\right]-K_{0}(\lambda H)\left[\beta K_{0}(H)-\alpha H K_{1}(H)\right]}
\end{aligned}
$$




$$
C_{4}=\frac{I_{0}(\lambda M)\left[\gamma I_{0}(\lambda H) K_{0}(H)-\gamma K_{0}(\lambda H) I_{0}(H)\right]}{I_{0}(\lambda H)\left[\beta K_{0}(H)-\alpha H K_{1}(H)\right]-K_{0}(\lambda H)\left[\beta K_{0}(H)-\alpha H K_{1}(H)\right]}
$$

\section{Nomenclature}

$r^{\prime} \quad$ dimensional radial coordinate

$u^{\prime} \quad$ axial velocity

$U \quad$ dimensionless axial velocity

$R \quad$ dimensionless radial coordinate

$T_{0} \quad$ reference temperature

$T_{w} \quad$ constant temperature

$\theta$ dimensionless temperature

a radius of the inner cylinder

$b$ radius of the outer cylinder

$B_{0} \quad$ constant magnetic flux density

$Q_{0} \quad$ dimensional heatgeneration/absorption coefficient

$g$ gravitational acceleration

$M \quad$ Hartmann number

$Q \quad$ dimensionless volume flow rate

$\mathrm{Pr} \quad$ Prandtl number

$C_{p} \quad$ specific heat at constant pressure

\section{Greek Letters}

$v$

fluid kinematic viscosity

$\tau \quad$ skin-friction

$\sigma \quad$ electrical conductivity of the fluid

$\rho$ density

$k \quad$ thermal conductivity of the fluid

$\lambda \quad$ radii $\operatorname{ratio}(b / a)$

$\beta \quad$ coefficient of thermal expansion

\section{References}

[1] S. Kakac, W. Aung and R. Viskanta eds., Natural Convection: Fundamentals and Applications, Hemisphere Publishing, New York, 1986.

[2] A, Bejan and A. D. Kraus, Heat transfer handbook, John Wiley \& Sons, Inc., Hoboken, New Jersey, 2003.

[3] J. S. Turner, Buoyancy Effects in Fluids, Cambridge University Press, Cambridge, 1973.

[4] R. M. Inman: Experimental study of temperature distribution in laminar tube flow of a fluid with internal heat generation, Int. J. Heat Mass Transfer, Vol. 5(11), 1053-1054, 1962.

[5] P. L. Chambre: The Laminar boundary layer with distributed heat sources or sinks, Appl. Sci. Res. Sec. A. Vol. 6(5), 393$401,1957$.

[6] J. Modejski: Temperature distribution in channel flow with friction, Int. J. Heat Mass Transfer Vol. 6(1), 49-51, 1963.

[7] H. L. Toor: The energy equation for viscous flow, Ind. Eng.
Chem. Vol. 48(5), 922-926, 1956.

[8] K. Vajravelu and J. Nayfeh: Hydromagnetic convection at a cone and a wedge. Int. Commun. Heat Mass Transfer, Vol. 19, 701-710, 1992.

[9] O. Miyatake and T. Fujii: Free convection heat transfer between vertical plates - one plate isothermally heated and other thermally insulated. Heat Transfer Japan Res. Vol.1, 30$38,1972$.

[10] H. Tanaka, O. Miyatake, T. Fujii and M. Fujii: Natural convection heat transfer between vertical parallel plates- one plate with a uniform heat flux and the other thermally insulated, Heat Transfer Japan Res. Vol. 2, 25-33, 1973.

[11] B. K. Jha and A. O. Ajibade: Unsteady free convective Couette flow of heat generating/absorbing fluid. Int. J. Energy and Tech. Vol. 2(12), 1-9, 2010.

[12] B. K. Jha and A. O. Ajibade: Free convection flow of heat generation/absorption fluid between vertical porous plates with periodic heat input. Int. comm. heat and mass transfer Vol. 36, 624-631, 2009.

[13] S. Das, B. C. Sarkar and R. N. Jana: Radiation effects on free convection MHD Couette flow started exponentially with variable wall temperature in presence of heat generation. Open J. Fluid Dynamics. Vol. 2, 14-27, 2012.

[14] C. Mandal, S. Das and R. N. Jana: Effect of radiation on transient natural convection flow between two vertical walls. Int. J. Appl. Inf. Systems. Vol. 2(2), 49-56, 2012.

[15] S. Das, S. K. Guchhait and R. N. Jana: Radiation effects on unsteady MHD free convective Couette flow of heat generation/absorbing fluid. Int. J. Com. Appl. Vol. 39(3), 42$51,2012$.

[16] A. J. Chamkha: hydromagnetics combined convection flow in a vertical lid-driven cavity with internal heat generation/absorption. Numer. Heat transfer A, Vol. 41, 529$546,2002$.

[17] S. K. Singh, B. K. Jha and A. K. Singh: Natural convection in vertical concentric annulus under a radial magnetic field. Heat and mass transfer Vol. 32, 399-401, 1997.

[18] B. Kalita: Unsteady free convection MHD flow and heat transfer between two heated vertical plates with heat source: an exact solution, J. Applied Mathematics \& Bioinformatics, Vol. 2(3), 1-15, 2012.

[19] R. K. Singh and A. K. Singh: Effect of induced magnetic field on natural convection in a vertical concentric annulus. Acta Mech. Sin. Vol. 28(2), 315-323, 2012.

[20] D. Kumar, A. K. Singh, Effects of heat source/sink and induced magnetic field on natural convective flow in vertical concentric annuli, Alexandria Eng. J. (2016), http://dx.doi.org/10.1016/j.aej.2016.08.019. 